\title{
Prognostic significance of exercise-induced premature ventricular complexes: a systematic review and meta-analysis of observational studies
}

\author{
Victor Lee, ${ }^{1}$ Dhanuka Perera, ${ }^{2}$ Pier Lambiase ${ }^{3}$
}

\begin{abstract}
- Additional material is available. To view please visit the journal online (http://dx. doi.org/10.1136/heartasia2016-010854)

${ }^{1}$ Princess Margaret Hospital, Kowloon, Hong Kong ${ }^{2}$ St Bartholomew's Medical School, London, UK ${ }^{3}$ University College London, London, UK
\end{abstract}

Correspondence to Dr Victor Lee, Princess Margaret Hospital, 2-10 Princess Margaret Hospital Road, Lai Chi Kok, Kowloon, Hong Kong;

victorkflee@hotmail.com

Received 25 October 2016 Accepted 3 November 2016

CrossMark

To cite: Lee $\mathrm{V}$, Perera $D$, Lambiase P. Heart Asia 2017;9:1-11. doi:10.1136/ heartasia-2016-010854

\section{ABSTRACT}

Background Exercise-induced premature ventricular complexes (EI-PVCS) are common during exercise stress tests. Their optimal management and prognostic significance remain uncertain.

Aim To perform meta-analysis of observational studies on the prognostic significance of EI-PVCs.

Methods A search was conducted on Medline and Embase. Inclusion criteria were observational studies comparing the prognosis of patients with and without EI-PVCs whilst exclusion criteria were studies without confounder adjustment and studies with zero endpoints. Composite endpoints included all-cause mortality, cardiac mortality and cardiovascular events. Relative risk of endpoints were analysed with random effects model. Meta-regression and sensitivity analysis were performed. Results Ten studies were included. In asymptomatic patients who had no clinical evidence of heart disease, EI-PVCs were associated with a pooled risk ratio of 1.82 (95\% Cl 1.44 to 2.30 ) of developing adverse cardiovascular events over 16 years. The corresponding pooled RR for patients with symptomatic heart disease was $1.36(95 \% \mathrm{Cl} 1.18$ to 1.57$)$ over 5.4 years.

Sensitivity analysis: only El-PVCs on the recovery phase of an exercise test, not during exercise, had adverse prognostic significance.

Conclusions EI-PVCs are correlated with a higher risk of all cause death or cardiovascular events in the long term. This risk is elevated in asymptomatic patients without clinical heart disease and in patients with symptomatic heart disease. The fact that only El-PVCs during recovery, and not during exercise, have poor prognostic value suggests that autonomic dysfunction may play a role in this association. Further studies are needed to see if autonomic manipulation by drugs or catheter-based methods can improve the poor prognosis associated with EI-PVCs.

\section{INTRODUCTION}

In the guidelines on the diagnosis and management of stable ischaemic heart disease issued by the American Heart Association and its allied societies, non-invasive assessment of myocardial ischaemia is the first line investigation for patients with low or intermediate pre-test probability of coronary artery disease. ${ }^{1}$ Exercise is a frequently used stressor in these tests. In addition to specified endpoints suggestive of ischaemia such as exercise-induced chest pain and ST segment deviation, exercise-induced premature ventricular complexes (EI-PVCs) commonly occur. The prevalence of EI-PVCs ranges from $7 \%$ in normal subjects ${ }^{2}$ to $54 \%$ in patients with cardiovascular disease. ${ }^{3}$ In regard to their prognostic significance, some studies reported an adverse prognosis associated with EI-PVCs ${ }^{4-13}$ while others found benign outcomes. ${ }^{14-33}$ These studies are difficult to interpret because they analyse different patient groups ranging from asymptomatic patients to patients with clinical heart disease such as heart failure and myocardial infarction. In addition, the majority of studies do not adjust for cardiovascular risk factors which can cause profound confounder bias. In order to guide clinicians on the management of EI-PVCs in these patients, we performed a systematic review and meta-analysis on studies looking at the prognosis of EI-PVCs, excluding unadjusted studies and stratified patients according to whether or not they have clinical heart disease.

\section{METHODS}

A systematic literature search was conducted on Medline (1965 to October 2016) and Embase (1974 to October 2016) for articles that examined the prognostic significance of EI-PVCs. Index terms used were 'Exercise' OR 'Treadmill' AND 'Ventricular' AND 'Arrhythmia' OR 'Premature Ventricular Complexes' OR 'Ectopic'. Further articles were obtained by hand-searching the reference lists of the relevant papers. A separate search for conference abstracts and proceedings was conducted on Embase using its advanced search option. It was not possible to identify studies that had neither been published nor presented at a conference because, unlike controlled trials, a registry for observational studies does not exist. There was no language restriction in our search.

A protocol for the meta-analysis was predetermined. The inclusion criterion for the meta-analysis was any observational study that compared the incidence of major adverse cardiac events or all cause death in patients with EI-PVCs to those without these arrhythmias. Exposure was simply the possession of EI-PVCs during the exercise stage or recovery stage of an exercise test. Outcomes were all cause mortality, cardiac mortality and cardiovascular events, namely non-fatal myocardial infarction, angina, cardiac hospital admissions and cardiac arrest. When different outcomes were analysed in the meta-analysis, only the all-cause mortality was used as an endpoint in the meta-analysis. We separately analysed asymptomatic patients without clinical heart disease and patients with symptomatic heart disease. Exclusion criteria were: (1) studies that did not adjust for cardiovascular risk factors, including age, gender, hypertension, diabetes; and (2) studies that reported zero endpoints in either 
group. Some studies examined exercise stage EI-PVCs and recovery EI-PVCs separately, for example, Frolkis et $a l^{33}$ and Dewey et $a l .{ }^{34}$ For the purpose of the meta-analysis, the separate substudies were counted as separate analyses. Likewise, some studies separately analysed patient groups with different EI-PVC frequencies. For the sake of this meta-analysis, these groups were also counted as different analyses. For example, in Morshedi-Meibodi's study, there were 399 patients with EI-PVCs $<0.22 / \mathrm{min}$ and 393 patients with EI-PVCs $>0.22 / \mathrm{min}$. These substudies were counted as separate analyses. In Dewey's study, not only were the EI-PVCs classified as exercise stage EI-PVCs and recovery EI-PVCs, they were also separated into frequent and non-frequent EI-PVCs. This study therefore generated four substudies for the meta-analysis.

\section{Data extraction for meta-analysis}

The full texts of the studies satisfying the inclusion criteria were obtained. For each study, the following data were extracted by the primary investigator: (1) clinical status: symptomatic status, presence of clinical heart disease; (2) patient characteristics: mean age, percentage male, percentage of patients with diabetes or hypertension, and proportion of patients with ischaemia detected on exercise test; (3) adjustment of confounders including age, gender, hypertension, diabetes, and ischaemia detected on exercise ECG; (4) follow-up data: study endpoints with their relative risks and 95\% CIs, and length of follow up.

\section{Statistical analysis}

Pooled relative risk (RR) of primary endpoints and their 95\% CIs were calculated using the random effects model. Meta-regression was used to investigate if any continuous variable (eg, percentage male, percentage with diabetes or hypertension, proportion of patients with ischaemic exercise ECG, length of follow-up) was associated with study outcomes. Sensitivity analysis was also performed to see if any categorical study characteristic (eg, whether ischaemia on exercise test or left ventricular (LV) function was adjusted for, stage of exercise test at which EI-PVCs occur) affected study endpoints. Studies that did not report specific data for a particular characteristic were excluded from the meta-regression and sensitivity analysis of that particular variable. Heterogeneity among the studies was evaluated using the $\mathrm{I} 2$ statistic. Publication bias was assessed by Egger's regression asymmetry test. All statistics were performed on Comprehensive Meta-Analysis software V.2, Biostat, Englewood, USA. The PRISMA (Preferred Reporting Items for Systematic reviews and Meta-Analyses) guidelines for the reporting of meta-analysis of observational studies in epidemiology were followed. ${ }^{35}$

\section{RESULTS}

The search strategy returned 3906 articles. There were no non-English articles identified. No relevant conference abstracts or proceedings were found. There were no potential conflicts of interest among the studies. Thirty-three articles were on the prognostic significance of EI-PVC; 23 were excluded according to our exclusion criteria (see online supplementary table S1).

Ten studies satisfied our inclusion criteria-four were on asymptomatic patients without clinical heart disease and six were on patients with symptomatic heart disease. In the asymptomatic without clinical heart disease group, Morshedi-Meibodi et $a l^{6}$ had two sub-studies separately analysing frequent and non-frequent EI-PVCs. This provided an extra analysis for this group, for example, a total of 5 analyses from 4 studies. On the other hand, in the group with symptomatic heart disease,
Frolkis et al separately analysed exercise stage EI-PVCs and recovery EI-PVCs. Dewey et $a l^{34}$ did the same and went further by dividing them into frequent and non-frequent EI-PVCs. Therefore, we have 10 analyses from 6 studies in this group.

Figure 1 shows the inclusion diagram for the meta-analysis. Table 1 lists all the studies that analysed asymptomatic patients without clinical evidence of heart disease, while table 2 shows all the studies of patients with symptomatic cardiac disease. A detailed description of each study is provided.

\section{Summary statistics}

In asymptomatic patients who had no clinical evidence of heart disease, EI-PVCs were associated with a pooled RR of 1.82 (95\% CI 1.44 to 2.30 ) of developing cardiovascular events (including non-fatal myocardial infarction, angina, cardiac hospital admissions, cardiac arrest), cardiovascular mortality and all cause mortality over a mean follow-up of 16 years. In patients with symptomatic heart disease, the corresponding pooled RR was 1.36 (95\% CI 1.18 to 1.57$)$ over a mean follow-up of 5.4 years. Figure 2 summarises the results as Forrest plots.

\section{Description of the studies and quality assessment}

Asymptomatic patients without clinical heart disease

There were four studies and five analyses in this group. ${ }^{4-6} 16$ They were all observational prospective cohort studies. The total number of patients studied was 1239, totalling 20150 patient-years of follow-up. The mean age was 47 years, and the mean sample size was 249 with an average male preponderance of $51 \%$. These studies ruled out clinical heart disease by history, physical examination and ECG with only one study using M-mode echocardiography. ${ }^{6}$ All adjusted for cardiovascular risk factor confounders-four analyses also controlled for ischaemia detected on exercise test ${ }^{5}{ }^{6}$ and nuclear perfusion scan, ${ }^{16}$ but no study controlled for LV function. Only one study provided data on LV function. ${ }^{6}$ One study ${ }^{6}$ examined the correlation of EI-PVC complexity and prognosis and found that, after multivariate analysis, it is not an independent risk factor of all-cause mortality (no point estimates were provided). None of the studies provided information on EI-PVC origin.

\section{Patients with symptomatic heart disease}

In this group, there were six studies $9212633 \quad 34$ and 10 analyses. The total number of patients was 4105, totalling 22080 patient years of follow-up. The mean age was 59 years, and the mean sample size was 410 with an average male preponderance of $87 \%$. This group analysed patients who had been referred for an exercise test due to chest pain, exertional dyspnoea, resting ECG abnormalities and exercise capacity assessment as well as post-myocardial infarction patients and heart failure patients. Seven were prospective cohort studies, ${ }^{9} 21263334$ and one was a retrospective analysis of prospective data. ${ }^{7}$ Among these patients, rest PVCs (defined as any PVC on resting ECG during $10 \mathrm{~s}$ before exercise) occurred in $0-29 \%$ of patients. EIPVC frequency ranged from $\geq 1$ PVC at any time to $>7$ PVCs at any stage to $10-20 \%$ of QRS. All the studies adjusted for confounders using Cox regression and propensity score matching techniques. Only four analyses had echocardiogram data and only two studies adjusted for LV function. ${ }^{21}{ }^{26}$ O'Neil et al ${ }^{26}$ studied whether EI-PVC complexity was associated with poor prognosis, and found that the complexity of EI-PVCs was associated with adverse events. ${ }^{26}$ Dewey et $a l^{34}$ studied the effect of EI-PVC frequency on its prognostic value, and found that both infrequent and frequent EI-PVCs are associated with poor prognosis. No studies provided data on EI-PVC origin. 


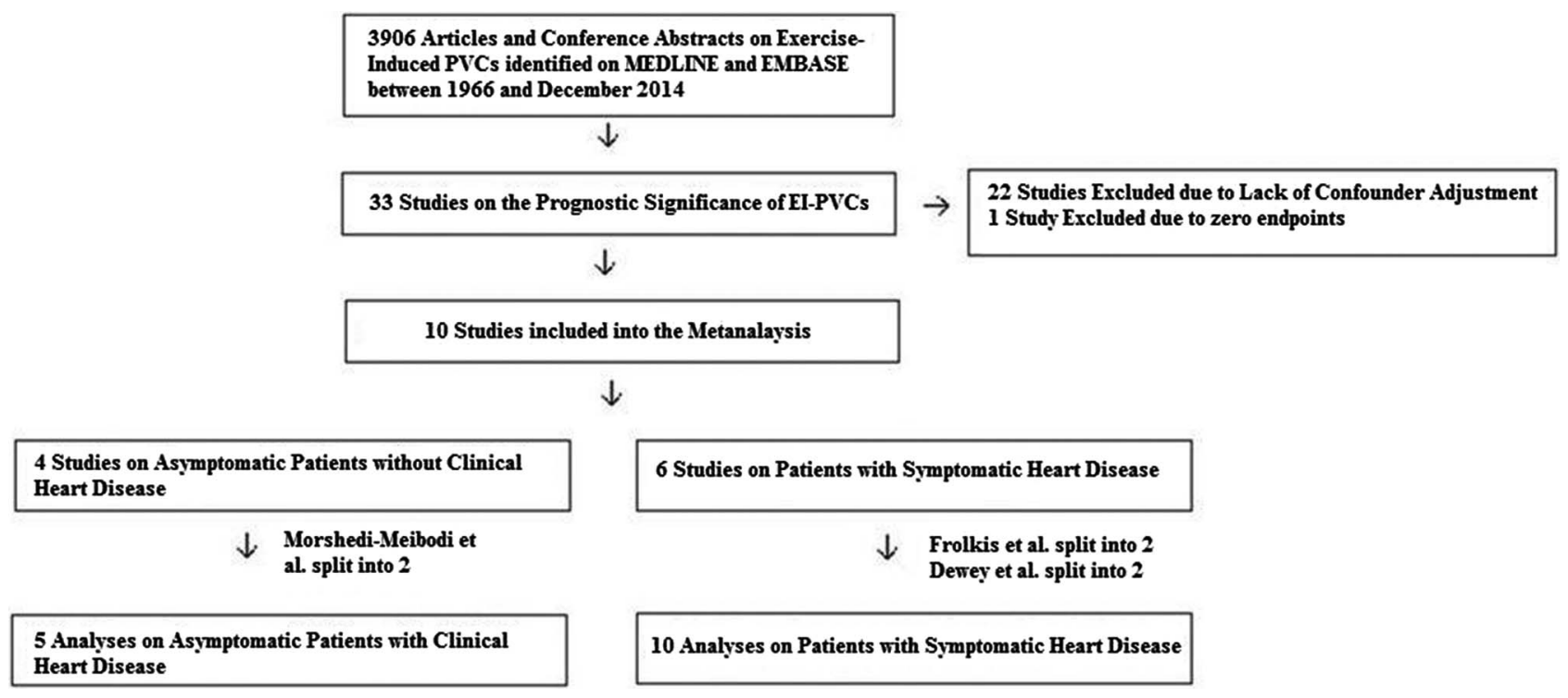

Figure 1 Inclusion diagram for meta-analysis. EI-PVC, exercise-induced premature ventricular complexes.

\section{Heterogeneity and publication bias}

The I2 statistic for the studies with and without clinical evidence of heart disease was 25 and 44, respectively, indicating only mild to moderate heterogeneity among these studies. The Egger's regression intercepts for studies with and without clinical evidence of cardiac disease were $-2.63(\mathrm{p}=0.2)$ and 1.72 $(\mathrm{p}=0.12)$, respectively, suggesting that there was no significant publication bias. Figure 3 shows the funnel plots for patients with and without clinical evidence of heart disease.

\section{Meta-regression and sensitivity analyses}

Asymptomatic patients without clinical heart disease

The only study in this group that showed a neutral prognosis for EI-PVCs was Bushby et al. Compared to the remaining studies, this was a very small study with short follow-up and therefore had limited power to pick up the adverse events associated with EI-PVCs. Meta-regression showed that there was a trend towards the length of study follow-up being correlated to study outcomes $(\mathrm{p}=0.06)$ (figure 4).

\section{Patients with symptomatic heart disease}

Our meta-regression showed that none of the study variablesmean age, $\%$ male, $\%$ with diabetes mellitus, $\%$ with hypertension, length of follow-up-correlated with endpoints. More importantly, there was no correlation between the percentage of patients with previous myocardial infarction and the percentage of patients with ischaemia on exercise test with study endpoints. The sensitivity analysis showed that: (1) adjustment for LV function and ischaemia detected on exercise test did not affect study outcomes; (2) types of study endpoints did not affect study outcomes either; (3) EI-PVCs occurring only during the exercise stage of the exercise test had no prognostic significance; only EI-PVCs that occurred during the recovery stage of the exercise test had adverse prognostic value (figure 5).

\section{DISCUSSION}

EI-PVCs are common in clinical practice. They are associated with increasing age, ${ }^{3}{ }^{29}$ male gender, ${ }^{36}$ cardiovascular risk factors such as blood pressure and fasting glucose, ${ }^{37}$ and structural heart disease. ${ }^{3} 1028$ Despite their prevalence, the optimal management of these arrhythmias is unclear. Studies on the prognostic significance of EI-PVCs have produced conflicting results. The studies in the literature are very heterogeneous and include patients ranging from asymptomatic athletes to heart failure patients and post-myocardial infarction patients. Furthermore, many studies did not make adjustment for cardiovascular risk factor confounders. We therefore performed a systematic review and meta-analysis on the long-term prognosis of EI-PVCs, excluding studies that did not adjust for cardiovascular risk factor confounders, and separately analysed asymptomatic patients without clinical evidence of heart disease and patients with symptomatic heart disease.

We have shown that EI-PVCs are associated with a higher risk of developing cardiovascular events (including non-fatal myocardial infarction, angina, cardiac hospital admissions, cardiac arrest), cardiovascular mortality and all cause mortality over the long term. The risk is elevated both in asymptomatic patients with no clinical evidence of heart disease and in patients with symptomatic heart disease.

One obvious explanation for such an association is that EI-PVCs might cause lethal arrhythmia. In Fioretti's study, ${ }^{21}$ EI-PVCs developed into ventricular fibrillation in a patient requiring defibrillation. This has been reported in $0.05 \%$ of exercise tests in the literature which means that 2000 patients need to be exercised to have one event of ventricular fibrillation. Since the mean sample size of our studies is 249 and 410, this cannot fully explain the association of EI-PVCs and poor prognosis found in our meta-analysis.

Another explanation can be the association of EI-PVCs with conditions that by themselves portend poor prognosis. EI-PVCs have been associated with LV dysfunction. ${ }^{2} \quad 10 \quad 28 \quad 30$ Nevertheless, our sensitivity analysis showed that the studies that adjusted for LV function did not find endpoints significantly different from those that did not adjust for LV function. However, as most studies in this meta-analysis did not assess LV function before enrolment, it is certainly possible that some patients with EI-PVCs have in fact undiagnosed cardiomyopathies such as hypertrophic cardiomyopathy or arrhythmogenic right ventricular dysplasia which by themselves have poorer prognosis. Similarly, EI-PVCs have been associated with ischaemia. $^{8} 293536$ Our meta-regression showed that the percentage of patients with ischaemia on exercise testing was not correlated 


\begin{tabular}{|c|c|c|c|c|c|c|c|c|c|c|c|c|}
\hline \multicolumn{13}{|c|}{ Patients without clinical evidence of cardiac disease } \\
\hline $\begin{array}{l}\text { Authors; } \\
\text { absence or } \\
\text { presence of } \\
\text { clinical heart } \\
\text { disease }\end{array}$ & $\begin{array}{l}\text { Subjects; } \\
\text { study type }\end{array}$ & $\begin{array}{l}\text { Exclusion } \\
\text { criteria; } \\
\text { reasons for } \\
\text { exercise test }\end{array}$ & $\begin{array}{l}\text { Total no. of } \\
\text { patients } \\
\text { with EI-PVC }\end{array}$ & $\begin{array}{l}\text { Exercise } \\
\text { protocol }\end{array}$ & $\begin{array}{l}\text { Mean } \\
\text { age; } \\
\% \\
\text { male }\end{array}$ & $\begin{array}{l}\text { Patient } \\
\text { characteristics; } \\
\text { mean EF/LV } \\
\text { function } \\
\text { assessment }\end{array}$ & $\begin{array}{l}\text { Confounder } \\
\text { adjustment; } \\
\text { statistics } \\
\text { method }\end{array}$ & $\begin{array}{l}\text { \% with } \\
\text { ischaemia } \\
\text { on exercise } \\
\text { test }\end{array}$ & $\begin{array}{l}\text { El-PVC definition; } \\
\text { EI-PVC } \\
\text { specification }\end{array}$ & End points & $\begin{array}{l}\text { Follow-up } \\
\text { (years); } \\
\text { lost to F/U }\end{array}$ & $\begin{array}{l}\text { RR of endpoints in } \\
\text { patients with EI-PVCs } \\
\text { compared to control } \\
\text { Assessment of bias } \\
\text { selection bias (SB) } \\
\text { confounder bias } \\
\text { (CB) } \\
\text { detection bias (DB) } \\
\text { attrition bias (AB) }\end{array}$ \\
\hline $\begin{array}{l}\text { Busby et } \mathrm{al}^{16} \\
\text { No clinical evidence } \\
\text { of heart disease }\end{array}$ & $\begin{array}{l}\text { Asymptomatic. } \\
\text { Absence of } \\
\text { cardiac disease by } \\
\text { history and ECG } \\
\text { Prospective cohort }\end{array}$ & $\begin{array}{l}\text { Clinical heart } \\
\text { disease. On } \\
\text { cardiac meds, } \\
\text { abnormal ECG }\end{array}$ & 80 & $\begin{array}{l}\text { Modified } \\
\text { Balke }\end{array}$ & $\begin{array}{l}64 \\
73 \%\end{array}$ & $\begin{array}{l}\text { 25\% HTN, } 22 \% \\
\text { Sm } 5 \% \text { DM, } \\
15 \% \text { lipid } \\
0 \% \text { Ml } \\
\text { Not available }\end{array}$ & $\begin{array}{l}\text { Ischaemic ET, } \\
\text { thallium } \\
\text { perfusion defect; } \\
\text { HTN, smoking, } \\
\text { DM, cholesterol, } \\
\text { age, gender } \\
\text { Age and gender } \\
\text { matched } \\
\text { control }\end{array}$ & $16 \%$ & $\begin{array}{l}\text { Frequent PVCs } \\
\text { defined as } 10 \% \text { of } \\
\text { all QRS complexes } \\
\text { in } 1 \text { min or VT } \\
\text { during and } \\
\text { post-exercise } \\
9 \% \text { had rest PVCs. } \\
\text { All had EI-PVCs } \\
>10 \% \text { of QRS; no } \\
\text { info on proportion } \\
\text { of recovery EI-PVCs. } \\
45 \% \text { had complex } \\
\text { EI-PVCs }\end{array}$ & $\begin{array}{l}\text { CV events } \\
\text { —angina } \\
\text { non-fatal } \\
\text { MI, } \\
\text { syncope }\end{array}$ & $\begin{array}{l}5.6 \\
2 \% \text { lost to } \\
\text { FU }\end{array}$ & $\begin{array}{l}0.78 \text { ( } 0.29 \text { to } 2.09) \\
\text { Adjusted } \\
\text { SB-No } \\
\text { CB-LV dysfunction } \\
\text { confounder } \\
\text { DB-CC } \\
\text { AB-CC }\end{array}$ \\
\hline $\begin{array}{l}\text { Jouven et al }{ }^{4} \\
\text { No clinical evidence } \\
\text { of heart disease }\end{array}$ & $\begin{array}{l}\text { Asymptomatic } \\
\text { patients free from } \\
\text { CAD } \\
\text { Prospective cohort }\end{array}$ & $\begin{array}{l}\text { Known or } \\
\text { suspected CVD, } \\
\text { BP }>180 \text {, } \\
\text { abnormal ECG }\end{array}$ & $\begin{array}{l}138 \\
\text { Hx free of } \\
\text { angina and } \\
\text { MI, ECG }\end{array}$ & $\begin{array}{l}\text { Bike } \\
\text { protocol }\end{array}$ & $\begin{array}{l}47 \\
100 \%\end{array}$ & $\begin{array}{l}1.4 \% \text { DM } \\
0 \% \text { MI } \\
\text { Not available }\end{array}$ & $\begin{array}{l}\text { Age, } \\
\text { blood pressure, } \\
\text { diabetes, } \\
\text { smoking } \\
\text { Cox regression }\end{array}$ & $6 \%$ & $\begin{array}{l}\text { Frequent EI-PVCs } \\
\text { defined as Run of } 2 \\
\text { or more or } 10 \% \text { of } \\
\text { all QRSs During and } \\
\text { post-exercise } \\
2.2 \% \text { had rest } \\
\text { PVCs, } 15 \% \\
\text { continued to have } \\
\text { PVCs during } \\
\text { recovery. No info on } \\
\text { PVC morphology }\end{array}$ & $\begin{array}{l}\text { CV } \\
\text { mortality }\end{array}$ & $\begin{array}{l}23 \\
5.5 \% \text { lost } \\
\text { to FU }\end{array}$ & $\begin{array}{l}2.53 \text { (1.65 to 3.88) } \\
\text { Adjusted } \\
\text { SB-No } \\
\text { CB-LV dysfunction as } \\
\text { possible confounder } \\
\text { DB-FU data reviewed } \\
\text { by independent } \\
\text { committee } \\
\text { AB-CC }\end{array}$ \\
\hline $\begin{array}{l}\text { Mora et } a l_{,}^{5} \\
\text { No clinical evidence } \\
\text { of heart disease }\end{array}$ & $\begin{array}{l}\text { Asymptomatic } \\
\text { patients free from } \\
\text { CAD } \\
\text { Prospective cohort }\end{array}$ & $\begin{array}{l}\text { Pregnant } \\
>30 \text { years, } \\
>80 \text { years, } \\
\text { angina, mi, } \\
\text { claudication, } \\
\text { stroke, LVH }\end{array}$ & $\begin{array}{l}229 \\
\mathrm{Hx} \text { free of } \\
\text { angina and } \\
\mathrm{MI}\end{array}$ & Bruce & $\begin{array}{l}52 \\
0 \%\end{array}$ & $\begin{array}{l}3 \% \text { DM, } 34 \% \text { Sm } \\
\text { Not available }\end{array}$ & $\begin{array}{l}\text { Age, HTN, DM, } \\
\text { smoking, } \\
\text { ischaemic ET } \\
\text { Cox regression }\end{array}$ & N/A & $\begin{array}{l}\text { El-PVCs defined as } \\
\text { multifocal or at } \\
\text { least } 10 \% \text { PVC in } \\
\text { last stage of } \\
\text { exercise or recovery } \\
\text { No info on rest } \\
\text { PVCs, recovery } \\
\text { PVCs, PVC } \\
\text { frequency or } \\
\text { morphology }\end{array}$ & $\begin{array}{l}\text { CV } \\
\text { mortality }\end{array}$ & $\begin{array}{l}20.3 \\
\text { No info }\end{array}$ & $\begin{array}{l}1.69 \text { (1.11 to2.58) } \\
\text { Adjusted } \\
\text { SB-No } \\
\text { CB-LV dysfunction, as } \\
\text { possible confounder } \\
\text { DB-2 readers } \\
\text { AB-CC }\end{array}$ \\
\hline
\end{tabular}


Table 1 Continued

Patients without clinical evidence of cardiac disease

\begin{tabular}{|c|c|c|c|c|c|c|c|c|c|c|c|c|}
\hline $\begin{array}{l}\text { Authors; } \\
\text { absence or } \\
\text { presence of } \\
\text { clinical heart } \\
\text { disease }\end{array}$ & $\begin{array}{l}\text { Subjects; } \\
\text { study type }\end{array}$ & $\begin{array}{l}\text { Exclusion } \\
\text { criteria; } \\
\text { reasons for } \\
\text { exercise test }\end{array}$ & $\begin{array}{l}\text { Total no. of } \\
\text { patients } \\
\text { with EI-PVC }\end{array}$ & $\begin{array}{l}\text { Exercise } \\
\text { protocol }\end{array}$ & $\begin{array}{l}\text { Mean } \\
\text { age; } \\
\% \\
\text { male } \\
\end{array}$ & $\begin{array}{l}\text { Patient } \\
\text { characteristics; } \\
\text { mean EF/LV } \\
\text { function } \\
\text { assessment }\end{array}$ & $\begin{array}{l}\text { Confounder } \\
\text { adjustment; } \\
\text { statistics } \\
\text { method }\end{array}$ & $\begin{array}{l}\% \text { with } \\
\text { ischaemia } \\
\text { on exercise } \\
\text { test }\end{array}$ & $\begin{array}{l}\text { EI-PVC definition; } \\
\text { EI-PVC } \\
\text { specification }\end{array}$ & End points & $\begin{array}{l}\text { Follow-up } \\
\text { (years); } \\
\text { lost to F/U }\end{array}$ & $\begin{array}{l}\text { Assessment of bias } \\
\text { - } \text { selection bias (SB) } \\
\text { confounder bias } \\
\text { (CB) } \\
\text { - detection bias (DB) } \\
\text { - attrition bias (AB) }\end{array}$ \\
\hline $\begin{array}{l}\text { Morshedi-Meibodi } \\
\text { et } \text { al }^{6} \\
\text { (Infrequent EI-PVC) } \\
\text { No clinical evidence } \\
\text { of heart disease }\end{array}$ & $\begin{array}{l}\text { Asymptomatic } \\
\text { patients free from } \\
\text { overt CAD } \\
\text { Prospective cohort }\end{array}$ & $\begin{array}{l}\text { CVD, valvular } \\
\text { heart disease, } \\
\text { COPD }\end{array}$ & $\begin{array}{l}399 \\
\text { Hx free of } \\
\text { angina and } \\
\text { Ml, physical } \\
\text { exam, ECG, } \\
\text { M-mode } \\
\text { echo }\end{array}$ & Bruce & $\begin{array}{l}44 \\
54 \%\end{array}$ & $\begin{array}{l}17 \% \text { HTN, } 3 \% \\
\text { DM, normal FS } \\
99.5 \%, 0 \% \mathrm{MI} \\
99.5 \% \text { have } \\
\text { normal fractional } \\
\text { shortening }\end{array}$ & $\begin{array}{l}\text { Age, HTN, } \\
\text { smoking, DM, } \\
\text { ischaemic ET, } \\
\text { complexity of } \\
\text { El-PVCs, LV } \\
\text { function } \\
\text { Cox regression }\end{array}$ & $8 \%$ & $\begin{array}{l}\text { Infrequent EI-PVCs } \\
\text { defined as }<0.22 \\
\text { El-PVC/min During } \\
\text { and post-exercise } \\
0 \% \text { had rest PVC, } \\
22 \% \text { had PVC } \\
\text { during recovery, } \\
\text { multifocal, 3\% } \\
\text { couplets; } 4 \%, 0 \% \\
\text { VT }\end{array}$ & $\begin{array}{l}\text { All cause } \\
\text { mortality }\end{array}$ & $\begin{array}{l}15 \\
2 \text { were lost } \\
\text { to FU }\end{array}$ & $\begin{array}{l}1.86 \text { (1.24 to 2.79) } \\
\text { Adjusted } \\
\text { SB-No } \\
\text { CB-Possible } \\
\text { undetected silent } \\
\text { coronary disease, } \\
\text { channelopathy, } \\
\text { hypertensive heart } \\
\text { DB-3 readers } \\
\text { AB-CC }\end{array}$ \\
\hline $\begin{array}{l}\text { Morshedi-Meibodi } \\
\text { et } \text { al }^{6} \\
\text { (frequent El-PVC) } \\
\text { No clinical evidence } \\
\text { of heart disease }\end{array}$ & $\begin{array}{l}\text { asymptomatic } \\
\text { patients free from } \\
\text { overt CAD } \\
\text { Prospective cohort }\end{array}$ & $\begin{array}{l}\text { CVD, valvular } \\
\text { heart disease, } \\
\text { COPD }\end{array}$ & $\begin{array}{l}393 \\
\text { Hx free of } \\
\text { angina and } \\
\text { Ml, physical } \\
\text { exam, ECG, } \\
\text { M-mode } \\
\text { echo }\end{array}$ & Bruce & $\begin{array}{l}47 \\
56 \%\end{array}$ & $\begin{array}{l}28 \% \text { HTN, } 3 \% \\
\text { DM, normal FS } \\
99.2 \%, 0 \% \mathrm{Ml} \text {, } \\
99.8 \% \text { have } \\
\text { normal fractional } \\
\text { shortening }\end{array}$ & $\begin{array}{l}\text { Age, HTN, } \\
\text { smoking, DM, } \\
\text { ischaemic ET, } \\
\text { complexity of } \\
\text { El-PVCs, LV } \\
\text { function } \\
\text { Cox regression }\end{array}$ & $9 \%$ & $\begin{array}{l}\text { Frequent EI-PVCs } \\
\text { defined as }>0.22 \\
\text { EI-PVC/min During } \\
\text { and post-exercise } \\
0 \% \text { had rest PVC, } \\
44 \% \text { had PVC } \\
\text { during recovery; } \\
22 \% \text { multifocal, } \\
17 \% \text { couplets, } 3 \% \\
\text { VT }\end{array}$ & $\begin{array}{l}\text { All cause } \\
\text { mortality }\end{array}$ & $\begin{array}{l}15 \\
2 \text { were lost } \\
\text { to FU }\end{array}$ & $\begin{array}{l}1.71 \text { (1.18-2.49) } \\
\text { Adjusted } \\
\text { SB-No } \\
\text { CB-Possible } \\
\text { undetected silent } \\
\text { coronary disease, } \\
\text { channelopathy, } \\
\text { hypertensive heart } \\
\text { DB-3 readers } \\
\text { AB-CC }\end{array}$ \\
\hline
\end{tabular}

$\mathrm{AB}$, attrition bias; $\mathrm{AF}$, atrial fibrillation; Angio, angiogram; $\mathrm{CABG}$, coronary artery bypass graft operation; CAD, coronary artery disease; CCF, congestive cardiac failure; $C V$, cardiovascular; $C B$, confounder bias; $C V D$, cardiovascular disease; $C V R F$, cardiovascular risk factors; COPD, chronic obstructive pulmonary disease; CXR, chest X-ray; DB, detection bias; DM, diabetes mellitus; Def, definition; Echo, echocardiogram; EF, ejection fraction; ET, exercise test; ESRF, end stage renal failure; Freq, frequency; HTN, hypertension; Info, information; Hx, history; Info, information; LVH, left ventricular hypertrophy; Meds, medications; MI, myocardial infarction; MPS, myocardial perfusion SPECT; PVC, premature ventricular complex; N, number; NS, not specified; PCI, percutaneous coronary intervention; Sm, smoker; SB, selection bias; TVD, triple vessel disease; VHD, valvular heart disease; VT, ventricular tachycardia. 
Table 2 Summary of all studies that included patients with clinical heart disease used in the meta-analysis

Patients with clinical evidence of cardiac disease

RR of endpoints in

patients with EI-PVCs

compared to control

Authors;

absence or

presence of

clinical heart Subjects;

disease study type

Exclusion criteria; Total no. of

Patient

characteristics; Confounder \% with

Assessment of bias

Beckerman High risk profile, $8.6 \%$ CABG,

$21 \% \mathrm{Ml}$,

$42 \%$ angina

Total no.

Mean maracteristics;
mean EF/LV

Confounder with

$\begin{array}{lll}\text { exercise test with EI-PVC } & \text { proto-col }\end{array}$

statistics

ischaemia

AF $\quad 42$

$77 \%$ for chest pain

$426 \quad$ Ramp

$63.6-51 \%$ Hin, $24 \%$
test

or ECG

abnormalities; $5 \%$

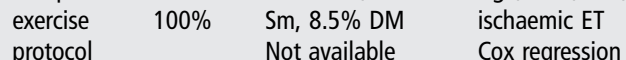

Prospective cohort for exercise

capacity

assessment, $2 \%$

for $\mathrm{SOB}$

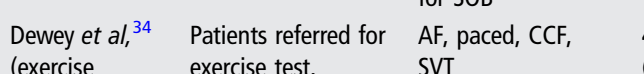

EI-PVCs)

11.6\% $\mathrm{Ml}, 11.1 \%$ No info

(frequent)

419

$\begin{array}{lll}\text { Ramp } & 56 & 22 \\ \text { protocol } & 97 \% & \text { DN } \\ & & \text { Not }\end{array}$

$22 \% \mathrm{Sm}, 15.2 \%$

I) Frequent $\quad \mathrm{PCl}, 39 \%$ chest

(infrequent)

Not available

Age,
DM

pain

Prospective cohort

\begin{tabular}{|c|c|c|}
\hline $\begin{array}{l}\text { Dewey et al, } \\
\text { (recovery } \\
\text { EI-PVCs) } \\
\text { I) Frequent } \\
\text { II) Infrequent }\end{array}$ & $\begin{array}{l}\text { Patients referred for } \\
\text { exercise test. } \\
15.1 \% \mathrm{Ml}, 15.1 \% \\
\mathrm{PCl} .40 \% \text { chest } \\
\text { pain } \\
\text { Prospective cohort }\end{array}$ & $\begin{array}{l}\text { AF, paced, CCF, } \\
\text { SVT } \\
\text { No info }\end{array}$ \\
\hline
\end{tabular}

\begin{tabular}{|c|c|c|c|}
\hline $\begin{array}{l}\text { Ramp } \\
\text { protocol }\end{array}$ & $\begin{array}{l}57 \\
96 \%\end{array}$ & $\begin{array}{l}29 \% \text { Sm, } 15 \% \\
\text { DM } \\
\text { Not available }\end{array}$ & $\begin{array}{l}\text { Age, Gender, } \\
\text { Diabetes } \\
\text { Propensity } \\
\text { Score } \\
\text { Matching }\end{array}$ \\
\hline
\end{tabular}

$6.8 \%$

Fioretti et $a^{21} \quad$ Pre-discharge,

post-MI

Heart failure,

32

Bicycle $\quad 59 \quad 100 \% \mathrm{Ml}$,

$\begin{array}{lll}59 & 100 \% \mathrm{Ml}, & \begin{array}{l}\text { Age, gender. } \\ \text { ischaemic } \mathrm{ET}\end{array}\end{array}$

$55 \%$

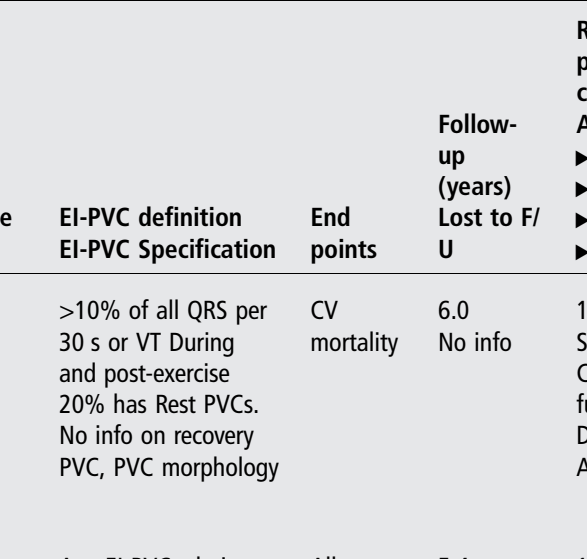

selection bias (SB)

confounder bias (CB)

- attrition bias (AB)

1.6 (1.1 to 2.3$)$

CB-Possible as no LV

function assessment

Any El-PVCs during

All cause $\quad 5.4$

1.34 (0.88 to 2.04$)$ for

stage of test

(frequent EI-PVC

defined as above

mean frequency-

$0.6 / \mathrm{min}$; infrequent

defined as below

mean frequency)

No specific info on

rest PVC

$0 \%$ had recovery

PVCs

No info on origin or

complexity

Any El-PVCs during

exercise or recovery

stage of test

(frequent EI-PVC

defined as above

mean frequency,

infrequent defined as

below mean

frequency)

No specific info on

rest PVC

$100 \%$ had PVCs in

recovery

No info on origin or

complexity

$>1$ EI-PVC at any

$$
\text { test, }
$$

$\begin{array}{ll}\text { All cause } & 5.4 \\ \text { mortality } & \text { No Info } \\ & \\ & \\ & \\ & \\ \text { CV } & 1.0 \\ \text { mortality } & 3 \text { lost to } \\ & \text { F/U }\end{array}$

1.71 (1.07 to 2.73 ) for infrequent

$\mathrm{SB}-\mathrm{No}$

CB-LV dysfunction as

possible confounder

DB-No; 2 observers

were blinded to exercise

$\mathrm{AB}-\mathrm{CC}$

2.3 (0.9 to 5.7 )

$\mathrm{SB}$-No

CB-Possible unknown

Continued

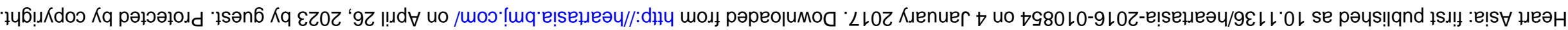


Table 2 Continued

Patients with clinical evidence of cardiac disease

\begin{tabular}{|c|c|c|c|c|c|c|c|c|c|c|c|c|}
\hline $\begin{array}{l}\text { Authors; } \\
\text { absence or } \\
\text { presence of } \\
\text { clinical heart } \\
\text { disease }\end{array}$ & $\begin{array}{l}\text { Subjects; } \\
\text { study type }\end{array}$ & $\begin{array}{l}\text { Exclusion criteria; } \\
\text { reasons for } \\
\text { exercise test }\end{array}$ & $\begin{array}{l}\text { Total no. of } \\
\text { patients } \\
\text { with EI-PVC }\end{array}$ & $\begin{array}{l}\text { Exercise } \\
\text { proto-col }\end{array}$ & $\begin{array}{l}\text { Mean } \\
\text { age; \% } \\
\text { male }\end{array}$ & $\begin{array}{l}\text { Patient } \\
\text { characteristics; } \\
\text { mean EF/LV } \\
\text { function } \\
\text { assessment }\end{array}$ & $\begin{array}{l}\text { Confounder } \\
\text { adjustment; } \\
\text { statistics } \\
\text { method }\end{array}$ & $\begin{array}{l}\% \text { with } \\
\text { ischaemia } \\
\text { on exercise } \\
\text { test }\end{array}$ & $\begin{array}{l}\text { EI-PVC definition } \\
\text { EI-PVC Specification }\end{array}$ & $\begin{array}{l}\text { End } \\
\text { points }\end{array}$ & $\begin{array}{l}\text { Follow- } \\
\text { up } \\
\text { (years) } \\
\text { Lost to F/ } \\
\text { U }\end{array}$ & $\begin{array}{l}\text { compared to control } \\
\text { Assessment of bias } \\
\text { selection bias (SB) } \\
\text { confounder bias (CB) } \\
\text { detection bias (DB) } \\
\text { attrition bias (AB) }\end{array}$ \\
\hline & & & & & & & & & $\begin{array}{l}0 \% \text { during recovery, } \\
50 \% \text { had rest PVCs } \\
>5 \text { min on } 24 \text { hour } \\
\text { Holter } \\
\text { No info on PVC } \\
\text { frequency, } \\
\text { morphology }\end{array}$ & & & $\begin{array}{l}\text { confounder } \\
\text { DB-CCAB-CC }\end{array}$ \\
\hline $\begin{array}{l}\text { Frolkis } \\
\text { et al, }{ }^{33} \\
\text { (during } \\
\text { exercise only) }\end{array}$ & $\begin{array}{l}\text { Patients referred for } \\
\text { exercise test, } 49 \% \\
\text { CAD, } 21 \% \text { MI } \\
\text { Retrospective } \\
\text { analysis of } \\
\text { prospective data }\end{array}$ & $\begin{array}{l}<30 \text { years of age, } \\
\text { heart failure, } \\
\text { valvular disease, } \\
\text { ESRF, AF } \\
\text { No info }\end{array}$ & 945 & NS & $\begin{array}{l}56 \\
81 \%\end{array}$ & $\begin{array}{l}13 \% \text { DM, } 45 \% \\
\text { HTN } \\
18 \% \text { had EF } \\
<40 \%\end{array}$ & $\begin{array}{l}\text { Age, gender, } \\
\text { DM, HTN. } \\
\text { ischaemic ET } \\
\text { Propensity } \\
\text { score } \\
\text { matching }\end{array}$ & $17 \%$ & $\begin{array}{l}\text { Frequent EI-PVCs } \\
\text { defined as } 7 \text { or more } \\
\text { PVCs/min or complex } \\
\text { during exercise } \\
\text { All had }>7 \text { PVC/min, } \\
0 \% \text { had PVC during } \\
\text { recovery } \\
\text { No info on rest PVC, } \\
1 \% \text { had bigeminy, } \\
0.5 \% \text { trigeminy, } 0.3 \% \\
\text { couplets, } 1 \% \text { triplets, } \\
0.01 \% \text { VT }\end{array}$ & $\begin{array}{l}\text { All cause } \\
\text { mortality }\end{array}$ & $\begin{array}{l}5.3 \\
\text { No info }\end{array}$ & $\begin{array}{l}1.1 \text { (0.9 to } 1.3 \text { ) } \\
\text { SB--No } \\
\text { CB-Possible unknown } \\
\text { confounder e.g. } \\
\text { channelopathy, } \\
\text { cardiomyopathy } \\
\text { DB-Possible } \\
\text { AB-CC }\end{array}$ \\
\hline $\begin{array}{l}\text { Frolkis } \\
\text { et al } l^{2} \\
\text { (Recovery) }\end{array}$ & $\begin{array}{l}\text { Patients referred for } \\
\text { exericise test. } 50 \% \% \\
\text { CAD, } 31 \% \mathrm{MI} \\
\text { Retrospective } \\
\text { analysis of } \\
\text { prospective data }\end{array}$ & $\begin{array}{l}<30 \text { years of age, } \\
\text { heart failure, } \\
\text { valvular disease, } \\
\text { ESRF, AF } \\
\text { No info }\end{array}$ & 589 & NS & $\begin{array}{l}61 \\
79 \%\end{array}$ & $\begin{array}{l}13 \% \text { DM, } 45 \% \\
\text { HTN, 49\% CAD, } \\
21 \% \text { Ml } \\
27 \% \text { had EF } \\
<40 \%\end{array}$ & $\begin{array}{l}\text { Age, gender, } \\
\text { DM, HTN, } \\
\text { ischaemic ET } \\
\text { Propensity } \\
\text { score matching }\end{array}$ & $14 \%$ & $\begin{array}{l}\text { Frequent EI-PVCs } \\
\text { defined as } 7 \text { or more } \\
\text { PVCs/min or complex } \\
\text { During exercise } \\
\text { all had }>7 \text { PVC/min, } \\
100 \% \text { had PVC } \\
\text { during recovery } \\
\text { No info on rest PVC, } \\
1 \% \text { had bigeminy, } \\
0.5 \% \text { trigeminy, } 0.2 \% \\
\text { couplets, } 0.5 \% \\
\text { triplets, } 0.3 \% \text { VT }\end{array}$ & $\begin{array}{l}\text { All cause } \\
\text { mortality }\end{array}$ & $\begin{array}{l}5.3 \\
\text { No info }\end{array}$ & $\begin{array}{l}1.5 \text { (1.1 to } 1.9) \\
\text { SB--No } \\
\text { CB-Possible unknown } \\
\text { confounder, eg, LVH, } \\
\text { channelopathy, } \\
\text { cardiomyopathy } \\
\text { DB-Possible } \\
\text { AB-CC }\end{array}$ \\
\hline$O^{\prime}$ Neill et $a l^{26}$ & $\begin{array}{l}\mathrm{EF}<35 \% \text {, } \\
50 \% \text { with known } \\
\text { CAD, 35\% MI, } \\
29 \% \text { CABG } \\
\text { Prospective cohort }\end{array}$ & $\begin{array}{l}\text { Age }<20, \text { ESRF, } \\
\text { VHD, cardiac } \\
\text { transplants }\end{array}$ & 140 & Naughton & $\begin{array}{l}57 \\
87 \%\end{array}$ & $\begin{array}{l}\text { DM } 36 \%, 49 \% \\
\text { HTN, 39\% } \\
\text { previous } \mathrm{Ml}, \\
\text { mean EF of } 16 \%\end{array}$ & $\begin{array}{l}\text { Age, CVRF, } \\
\text { LV function }\end{array}$ & Not available & $\begin{array}{l}\text { Complex and frequent } \\
\text { El-PVCs during } \\
\text { recovery } \\
\text { (frequent El-PVC } \\
\text { defined as }>7 / m i n \\
\text { during recovery, } \\
\text { complex EI-PVCS } \\
\text { defined as couplets, } \\
\text { VT, bigeminy or } \\
\text { trigeminy) } \\
29 \% \text { had PVC at rest. } \\
100 \% \text { had PVC }\end{array}$ & $\begin{array}{l}\text { All cause } \\
\text { mortality }\end{array}$ & $\begin{array}{l}2.9 \\
\text { No Info }\end{array}$ & $\begin{array}{l}1.43 \text { (1.03 to } 1.99) \\
\text { Non-complex frequent El } \\
\text { - PVCS were not } \\
\text { associated with worse } \\
\text { prognosis } \\
\text { SB-No } \\
\text { CB-Possible unknown } \\
\text { confounder e.g. } \\
\text { channelopathy, } \\
\text { cardiomyopathy } \\
\text { DB-CC } \\
\text { AB-CC }\end{array}$ \\
\hline
\end{tabular}


Table 2 Continued

Patients with clinical evidence of cardiac disease

\begin{tabular}{|c|c|c|c|c|c|c|c|c|c|c|c|c|}
\hline $\begin{array}{l}\text { Authors; } \\
\text { absence or } \\
\text { presence of } \\
\text { clinical heart } \\
\text { disease }\end{array}$ & $\begin{array}{l}\text { Subjects; } \\
\text { study type }\end{array}$ & $\begin{array}{l}\text { Exclusion criteria; } \\
\text { reasons for } \\
\text { exercise test }\end{array}$ & $\begin{array}{l}\text { Total no. of } \\
\text { patients } \\
\text { with EI-PVC }\end{array}$ & $\begin{array}{l}\text { Exercise } \\
\text { proto-col }\end{array}$ & $\begin{array}{l}\text { Mean } \\
\text { age; } \% \\
\text { male }\end{array}$ & $\begin{array}{l}\text { Patient } \\
\text { characteristics; } \\
\text { mean EF/LV } \\
\text { function } \\
\text { assessment }\end{array}$ & $\begin{array}{l}\text { Confounder } \\
\text { adjustment; } \\
\text { statistics } \\
\text { method }\end{array}$ & $\begin{array}{l}\% \text { with } \\
\text { ischaemia } \\
\text { on exercise } \\
\text { test }\end{array}$ & $\begin{array}{l}\text { EI-PVC definition } \\
\text { EI-PVC Specification }\end{array}$ & $\begin{array}{l}\text { End } \\
\text { points }\end{array}$ & $\begin{array}{l}\text { Follow- } \\
\text { up } \\
\text { (years) } \\
\text { Lost to F/ } \\
\text { U }\end{array}$ & $\begin{array}{l}\text { RR of endpoints in } \\
\text { patients with EI-PVCs } \\
\text { compared to control } \\
\text { Assessment of bias } \\
\text { selection bias (SB) } \\
\text { confounder bias (CB) } \\
\text { detection bias (DB) } \\
\text { attrition bias (AB) }\end{array}$ \\
\hline & & & & & & & & & $\begin{array}{l}\text { during recovery. No } \\
\text { info on PVC origin. }\end{array}$ & & & \\
\hline $\begin{array}{l}\text { Partington } \\
\text { et } a l^{9}\end{array}$ & $\begin{array}{l}\text { Referred for ET, } \\
36 \% \mathrm{MI}, 13 \% \\
\text { CABG } \\
\text { Prospective cohort }\end{array}$ & $\begin{array}{l}\text { None } \\
\text { No info }\end{array}$ & 503 & $\begin{array}{l}\text { Not } \\
\text { available }\end{array}$ & $\begin{array}{l}64 \\
77 \%\end{array}$ & $\begin{array}{l}50 \% \text { HTN, } 24 \% \\
\text { Sm, } 9 \% \text { DM, } \\
13 \% \text { CABG, } 36 \% \\
\text { MI } \\
\text { Not available }\end{array}$ & $\begin{array}{l}\text { Age, CVRF, } \\
\text { Ischaemic ET } \\
\text { Cox regression }\end{array}$ & $35 \%$ & $\begin{array}{l}\text { Frequent El-PVCs } \\
\text { defined as }>10 \% \text { of } \\
\text { all QRS during } 30 \mathrm{~s} \text { or } \\
\text { VT During and } \\
\text { post-exercise } \\
20 \% \text { had rest PVC. } \\
\text { No info on PVC } \\
\text { morphology or } \\
\text { recovery PVC or LV } \\
\text { function }\end{array}$ & $\begin{array}{l}\text { All cause } \\
\text { mortality }\end{array}$ & $\begin{array}{l}6.0 \\
\text { No Info }\end{array}$ & $\begin{array}{l}1.24 \text { (1.03 to } 1.49) \\
\text { SB-No } \\
\text { CB-Possible unknown } \\
\text { confounder eg, LV } \\
\text { function } \\
\text { DB-CC } \\
\text { AB-CC }\end{array}$ \\
\hline
\end{tabular}

$A B$, attrition bias; $A F$, atrial fibrillation; Angio, angiogram; $C A B G$, coronary artery bypass graft operation; $C A D$, coronary artery disease; $C B$, confounder bias; CC, cannot comment due to lack of information; $C C F$, congestive cardiac failure; COPD, chronic obstructive pulmonary disease; CVD, cardiovascular disease; CVRF, cardiovascular risk factors; CXR, chest X-ray; DB, detection bias; Def, definition; DM, diabetes mellitus; Echo, echocardiogram; EF, ejection fraction; ESRF, end stage renal failure; ET, exercise test; Freq, frequency; HTN, hypertension; Hx, history; Info, information; Info, information; LVH, left ventricular hypertrophy; Meds, medications; MI, myocardial infarction; MPS, myocardial perfusion SPECT; N, number; NS, not specified; PCI, percutaneous coronary intervention; PVC, premature ventricular complex; SB, selection bias; Sm, smoker; TVD, triple vessel disease; VHD, valvular heart disease; VT, ventricular tachycardia. 


\section{A Study name}

Asymptomatic Patients Without Clinical Heart Disease

Bushby et al., 1989

Jouven et al., 2000

Mora et al., 2003

Morshedi-Meibodi et al., 2004 (Frequent El-PVCs)

Morshedi-Meibodi et al., 2004 (Infrequent EI-PVCs)

Pooled RR

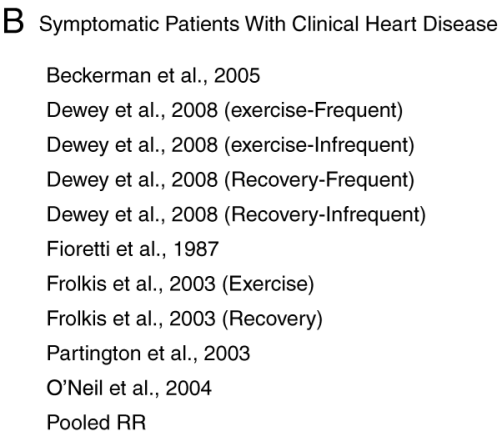

Statistics for each study

$\begin{array}{lccrr}\begin{array}{l}\text { Risk } \\ \text { Ratio }\end{array} & \begin{array}{c}\text { Lower } \\ \text { limit }\end{array} & \begin{array}{c}\text { Upper } \\ \text { limit }\end{array} & \text { Z-Value } & \text { p-Value } \\ 0.778 & 0.290 & 2.085 & -0.499 & 0.617 \\ 2.530 & 1.650 & 3.880 & 4.255 & 0.000 \\ 1.690 & 1.109 & 2.577 & 2.439 & 0.015 \\ 1.710 & 1.177 & 2.484 & 2.816 & 0.005 \\ 1.860 & 1.240 & 2.790 & 3.000 & 0.003 \\ 1.818 & 1.436 & 2.302 & 4.967 & 0.000\end{array}$

Risk Lower Upper

Ratio limit limit Z-Value $p$-Value

$\begin{array}{lllll}1.600 & 1.100 & 2.327 & 2.459 & 0.014\end{array}$

$\begin{array}{lllll}1.340 & 0.880 & 2.040 & 1.364 & 0.172\end{array}$

$\begin{array}{lllll}0.830 & 0.535 & 1.288 & -0.831 & 0.406\end{array}$

$\begin{array}{lllll}1.710 & 1.071 & 2.731 & 2.245 & 0.025\end{array}$

$\begin{array}{lllll}1.960 & 1.315 & 2.921 & 3.305 & 0.001\end{array}$

$\begin{array}{lllll}2.300 & 0.914 & 5.788 & 1.769 & 0.077\end{array}$

$\begin{array}{lllll}1.100 & 0.900 & 1.344 & 0.931 & 0.352\end{array}$

$\begin{array}{lllll}1.500 & 1.100 & 2.045 & 2.562 & 0.010\end{array}$

$\begin{array}{lllll}1.240 & 1.031 & 1.491 & 2.284 & 0.022\end{array}$

$\begin{array}{llllll}1.430 & 1.029 & 1.988 & 2.129 & 0.033\end{array}$

$\begin{array}{lllll}1.360 & 1.177 & 1.571 & 4.178 & 0.000\end{array}$

Risk Ratios and their $95 \% \mathrm{Cl}$

$\mid$

0.01

$\mid$

0.01

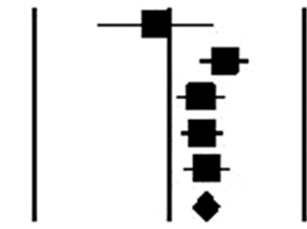

0.1

1

10

100
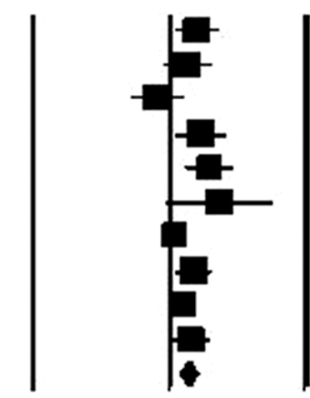

0.1

Decreased Endpoints

Figure 2 Forrest plots of studies stratified by whether or not patients have clinical heart disease.

with study outcomes, while our sensitivity analysis showed that adjusting for ischaemia detected on exercise testing did not affect study endpoints. In Jouven et al, ${ }^{4}$ only $3 \%$ of patients positive for ischaemia on exercise testing had EI-PVC and, conversely, only $6 \%$ of EI-PVC patients had a positive test for ischaemia. They found that exercise induced PVCs, independent of ischaemia, were associated with poor prognosis. ${ }^{4}$ Thus, another explanation behind the adverse prognostic effect of EI-PVCs is needed.

Our meta-regression shows that only EI-PVCs in the recovery period, not during exercise, were associated with poor prognosis. This suggests that EI-PVCs may be a manifestation of autonomic dysregulation which itself has been associated with increased mortality. Ducceschi et $a l^{38}$ showed that patients with EI-PVCs had longer corrected QT intervals at rest and during exercise than patients without EI-PVCs. Cole et $a l^{39}$ showed that decreased vagal reactivation post-exercise was associated with increased mortality independent of cardiac risk factors and myocardial perfusion abnormalities. The increased PVC burden in recovery could be due to increased sympathetic drive which itself could create a pro-arrhythmic substrate with increased conduction and repolarisation heterogeneities. Therefore, autonomic dysregulation may explain the poor prognosis associated with EI-PVCs.

Autonomic dysregulation could be a legitimate therapeutic target. This imbalance in autonomic tone can be modulated by pharmacological (eg, $\beta$-blockade), catheter-based and surgical strategies. Autonomic modulation, whether by pharmacological or catheter-based methods, is an exciting new frontier in electrophysiology. Further studies are required to see if such manipulation can reverse the adverse prognosis associated with EI-PVCs.

\section{Study limitations}

This meta-analysis is limited by various shortfalls inherent in observational studies, including unknown confounder effects and the inability to infer causality. Studies that have neither been presented nor published are missing. LV function assessment was rare in these studies; therefore patients with ventricular
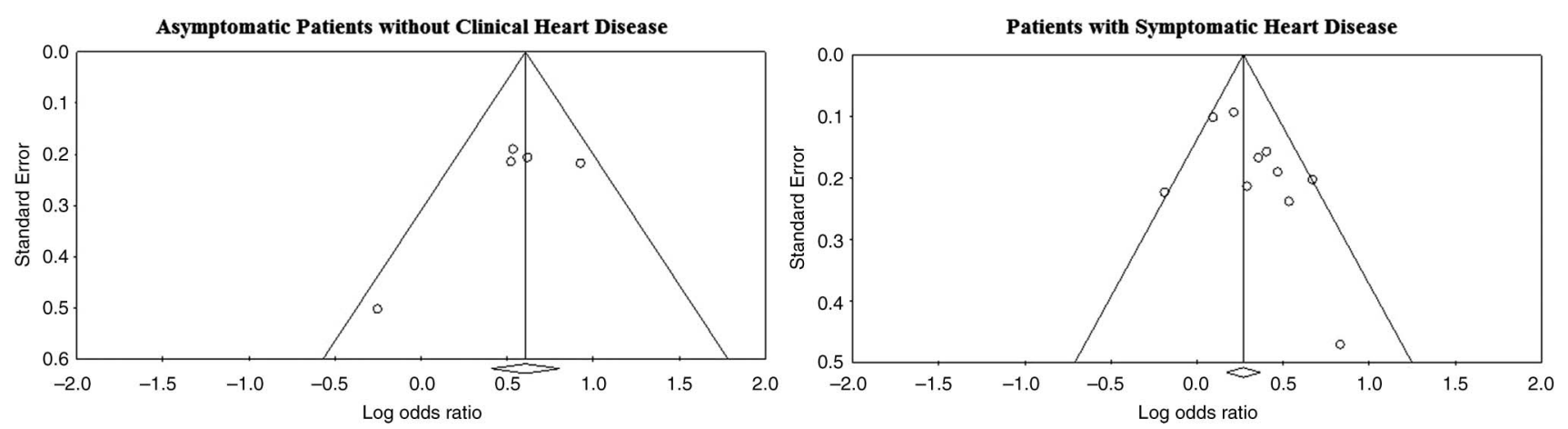

Figure 3 Funnel plots of the studies categorised by whether or not patients have clinical heart disease. 
hypertrophy, valvular heart disease or cardiomyopathy may have acted as confounders. In addition, patients with channelopathies could also have acted as possible confounders, although their effect probably is not significant due to their low prevalence. Although our I2 analysis showed freedom from severe heterogeneity, the heterogeneity was moderate and reflects the different demographics of the patients recruited in these studies. Likewise, although there is no significant publication bias, there was a trend towards publication bias in the studies that recruited patients with symptomatic heart disease. Nonetheless, this was adjusted with the Duvell and Tweedie's Trim and Fill method which still showed significant negative prognostic impact of EI-PVCs.

\section{CONCLUSIONS}

EI-PVCs, especially those that occur during recovery, are associated with a higher risk of death and cardiovascular events in
Figure 4 Meta-regression of mean length of follow up on log or of study outcomes.

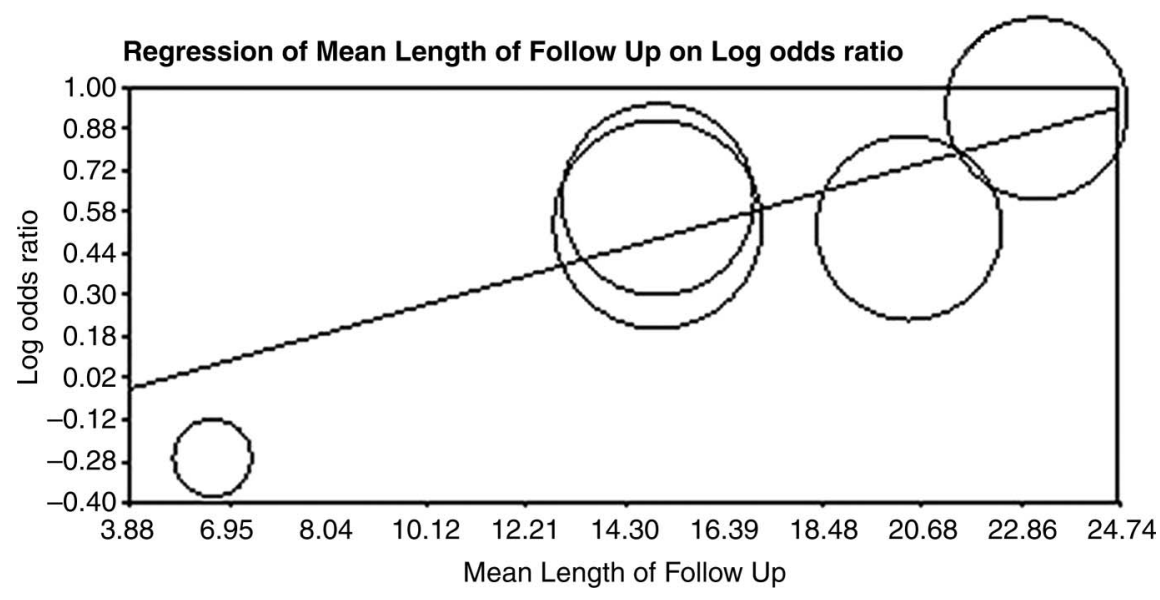

Risk Ratios and $95 \% \mathrm{Cl}$

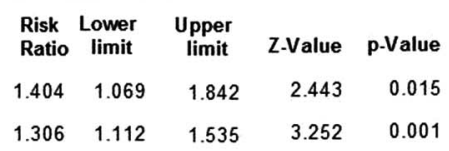

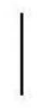

0.01

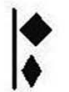

1

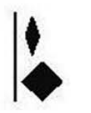

1

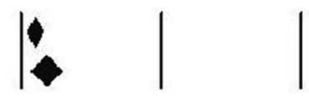

0.1

Decreased Endpoints

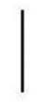

10

100
Controlled for LV

Function

No $\quad(N=8)$

Yes $(\mathbf{N}=\mathbf{2})$

Study Endpoints

$\begin{array}{lllllll}\text { All Cause Mortality } & (\mathrm{N}=8) & 1.319 & 1.132 & 1.539 & 3.536 & 0.000\end{array}$ $\begin{array}{lllllll}\text { Cardiovascular Mortality } & (\mathrm{N}=2) & 1.684 & 1.190 & \mathbf{2} .383 & 2.943 & 0.003\end{array}$

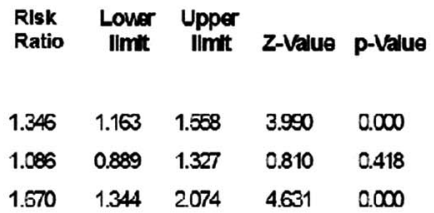

Pooled Risk Ratio and their $95 \% \mathrm{Cl}$

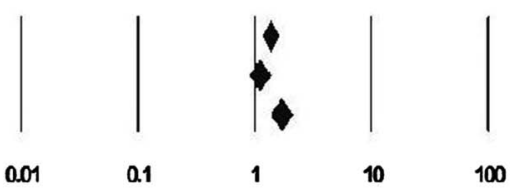

$\begin{array}{ll}\text { Decreased } & \text { Increased } \\ \text { Endpoints } & \text { Endpoints }\end{array}$

Figure 5 Sensitivity analysis of study endpoints according to whether ischaemia, left ventricular (LV) function, types of study endpoints and the stage at which exercise-induced premature ventricular complexes (EI-PVCS) appear during the exercise test. 
the long term. This risk is elevated both in asymptomatic patients without evidence of cardiac disease and in patients with symptomatic heart disease. Part of this correlation is due to its association with higher cardiovascular risk profile, cardiac ischaemia and undetected structural heart disease. Autonomic dysregulation may be an additional mechanism behind the poor prognostic effect of EI-PVCs. Patients who have EI-PVCs warrant further investigations to evaluate the cardiac function and coronary anatomy. If any abnormalities are detected, they should be managed accordingly. Further studies are needed to see whether autonomic manipulation either by pharmacological or catheter-based methods can improve the adverse prognostic effect of EI-PVCs.

Contributors VL conducted the data collection and data-analysis together with manuscript preparation. DP and PL reviewed the manuscript and provided important contributions to the manuscript.

Competing interests None declared.

Provenance and peer review Not commissioned; externally peer reviewed.

\section{REFERENCES}

1 Fihn SD, Gardin JM, Abrams J, et al. ACCF/AHA/ACP/AATS/PCNA/SCAI/STS guideline for the diagnosis and management of patients with stable ischemic heart disease. Executive Summary - a report of the American College of Cardiology Foundation Task Force on Practice Guidelines, and the American College of Physicians, American Association for Thoracic Surgery, Preventive Cardiovascular Nurses Association, Society for Cardiovascular Angiography and Interventions, and Society of Thoracic Surgeons. Circulation 2012;126:3097-137.

2 Verdile L, Maron BJ, Pelliccia A, et al. Clinical significance of exercise-induced ventricular tachyarrhythmias in trained athletes without cardiovascular abnormalities. Heart Rhythm 2015;12:78-85.

3 Goldschlager N, Cake D, Cohn K. Exercise-induced ventricular arrhythmias in patients with coronary artery disease. Their relation to angiographic findings. Am J Cardiol 1973;31:434-40.

4 Jouven $X$, Zureik $M$, Desnos $M$, et al. Long-term outcome in asymptomatic men with exercise-induced premature ventricular depolarizations. $N$ Eng J Med 2000:323:826-33.

5 Mora S, Redberg RF, Cui Y, et al. Ability of exercise testing to predict cardiovascular and all-cause death in asymptomatic women. a 20-year follow-up of the lipid research clinics prevalence study. JAMA 2003;290:1600-7.

6 Morshedi-Meibodi A, Evans JC, Levy D, et al. Clinical correlates and prognostic significance of exercise-induced ventricular premature beats in the community. The Framingham Heart Study. Circulation 2004;109:2417-22.

7 Beckerman J, Mathur A, Stahr S, et al. Exercise-induced ventricular arrhythmias and cardiovascular death. Ann Noninvasive Electrocardiol. 2005;10:47-52.

8 Elhendy A, Chandrasekaran K, Gersh BJ, et al. Functional and prognostic significance of exercise-induced ventricular arrhythmias in patients with suspected coronary artery disease. Am J Cardiol 2002;90:95-100.

9 Partington S, Myers J, Cho S, et al. Prevalence and prognostic value of exercise-induced ventricular arrhythmias. Am Heart J 2003;145:139-46.

10 Califf RM, McKinnis RA, McNeer JF, et al. Prognostic value of ventricular arrhythmias associated with treadmill exercise testing in patients studied with cardiac catheterization for suspected ischaemic heart disease. J Am Coll Cardiol 1983:2:1060-7.

11 Markiewicz W, Houston N, Debusk RF. Exercise testing soon after myocardial infarction. Circulation 1977;56:26-31.

12 Nair CK, Thomson W, Aronow WS, et al. Prognostic significance of exercise-induced complex ventricular arrhythmias in coronary artery disease with normal and abnormal left ventricular ejection fraction. Am J Cardiol 1984;54: $1136-8$.

13 Stone PH, Turi ZG, Muller JE, et al. Prognostic significance of the treadmill exercise test performance 6 months after myocardial infarction. J Am Coll Cardiol 1986;8:1007-17.

14 Weld FM, Chu KL, Bigger JT, et al. Risk stratification with low-level exercise testing 2 weeks after acute myocardial infarction. Circulation 1981;64:306-14.
15 Bruce RA, DeRouen TA, Hossack KF. Value of maximal exercise tests in risk assessment of primary coronary heart disease events in healthy men. Five years' experience of the Seattle heart watch study. Am I Cardiol 1980;46: 371-8.

16 Busby MJ, Shefrin EA, Fleg JL. Prevalence and long-term significance of exercise induced frequent or repetitive ventricular ectopic beats in apparently healthy volunteers. J Am Coll Cardiol 1989;14:1659-65.

17 Nair CK, Aronow WS, Sketch MH, et al. Diagnostic and prognostic significance of exercise-induced premature ventricular complexes in men and women: a four year follow-up. J Am Coll Cardiol 1983;1:1201-6.

18 Casella G, Pavesi PC, Sangiorgio P, et al. Exercise-induced ventricular arrhythmias in patients with healed myocardial infarction. Int J Cardiol 1993;40:229-35.

19 Debusk RF, Davidson DM, Houston N, et al. Serial ambulatory electrocardiography and treadmill exercise testing after uncomplicated myocardial infarction. $\mathrm{Am}$ J Cardiol 1980;45:547-54.

20 Ericsson M, Granath A, Ohlsen P, et al. Arrhythmias and symptoms during treadmill testing three weeks after myocardial infarction in 100 patients. Br Heart J 1973;35:787-90.

21 Fioretti P, Deckers J, Baardman T, et al. Incidence and prognostic implications of repetitive ventricular complexes during pre-discharge bicycle ergometry after myocardial infarction. Eur Heart J 1987;8(Suppl D):51-4.

22 Granath A, Sodermark T, Winge T, et al. Early work load tests for evaluation of long-term prognosis of acute myocardial infarction. Br Heart J 1973;35:758-63.

23 Henry RL, Kennedy GT, Crawford MH. Prognostic value of exercise-induced ventricular ectopic activity for mortality after acute myocardial infarction. Am J Cardiol 1987;59:1251-5.

24 Ivanova LA, Mazur NA, Smirnova TM, et al. Electrocardiographic exercise testing and ambulatory monitoring to identify patients with ischaemic heart disease at high risk of sudden death. Am J Cardiol 1980;45:1132-8.

25 Krone RJ, Gillespie JA, Weld FM, et al. Low-level exercise testing after myocardial infarction: usefulness in enhancing clinical risk stratification. Circulation 1985:71:80-9.

26 O'Neil JO, Young JB, Pothier CE, et al. Severe frequent ventricular ectopy after exercise as a predictor of death in patients with heart failure. J Am Coll Cardiol 2004:44:820-6

27 Peduzzi P, Hultgren $H$, Thomsen J, et al. Prognostic value of baseline exercise tests. Progress Cardiovasc Dis 1986:4:285-92.

28 Sami M, Chaitman B, Fisher L, et al. Significance of exercise-induced ventricular arrhythmia in stable coronary artery disease: a coronary artery surgery study project. Am J Cardiol 1984;54:1182-8.

29 Schweikert RA, Pashkow FJ, Snader CE, et al. Association of exercise-induced ventricular ectopic activity with thallium myocardial perfusion and angiographic coronary artery disease in stable, low-risk populations. Am J Cardiol 1999:83:530-4.

30 Weiner $\mathrm{DA}$, Levine $\mathrm{SR}$, Klein MD, et al. Ventricular arrhythmias during exercise testing: mechanism, response to coronary bypass surgery, and prognostic significance. Am J Cardiol 1984;53:1553-7.

31 De Paola AAV, Gomes JA, Terzian AB, et al. Ventricular tachycardia during exercise testing as a predictor of sudden death in patients with chronic chagasic cardiomyopathy and ventricular arrhythmias. Br Heart J 1995;74:293-5.

32 Yang JC, Wesley RC, Froelicher VF. Ventricular tachycardia during routine treadmill testing. Risk and prognosis. Arch Intern Med 1991;151:349-53.

33 Frolkis JP, Pothier CE, Blackstone EH, et al. Frequent ventricular ectopy after exercise as a predictor of death. N Eng J Med 2003;348:781-90.

34 Dewey FE, Kapoor JR, Williams RS, et al. Ventricular arrhythmias during clinical treadmill testing and prognosis. Arch Intern Med 2008;168:225-34.

35 Moher D, Liberati A, Tetzlaff J, et al., The PRISMA Group. Preferred reporting items for systematic reviews and meta-analyses: the PRISMA statement. Ann Intern Med 2009:151:264-9.

36 Marieb MA, Beller GA, Gibson RS, et al. Clinical relevance of exercise-induced ventricular arrhythmias in suspected coronary artery disease. Am I Cardiol 1990;66:172-8.

37 Vedin JA, Wilhelmsson CE, Wilhelmsen L, et al. Relation of resting and exercise-induced ectopic beats to other ischemic manifestations and to coronary risk factors. Men born in 1913. Am J Cardiol 1972;30:25-31.

38 Ducceschi V, Sarubbi B, Lucca P, et al. QTc and not QTc dispersion behaviour affects the occurrence of ventricular extrasystole during exercise in infarcted patients. Heart Vessels 1997:12:27-33.

39 Cole CR, Blackstone EH, Pashokow FJ, et al. Heart-rate recovery immediately after exercise as a predictor of mortality. N Engl J Med 1999;341:1351-7. 